\title{
Patient Safety and Preoperative Assessment of Neurological Status in Neurosurgical Patients
}

\section{Kemal Tolga Saracoglu*}

Department of Anesthesiology, Marmara University School of Medicine, Istanbul, Turkey

*Corresponding author: Kemal Tolga Saracoglu, Department of Anesthesiology, Marmara University School of Medicine, Fevzi Cakmak Mah. Mimar Sinan Cad, Pendik Istanbul, Turkey, Tel: +902166570606; E-mail: saracoglukt@gmail.com

Rec date: Jul 7, 2014; Acc date: Jul 8, 2014; Pub date: Jul 10, 2014

Copyright: @ 2014 Saracoglu KT. This is an open-access article distributed under the terms of the Creative Commons Attribution License, which permits unrestricted use, distribution, and reproduction in any medium, provided the original author and source are credited.

\section{Editorial}

Patients scheduled for neurosurgical procedures can have a wide range of symptoms. Neurosurgical patients range from awake and easy-going patients undergoing elective surgery to trauma patients scheduled for emergency surgery due to depressed neurological status. Therefore, a detailed preoperative assessment of physical and neurological status is important for generating a proper anesthesia plan for the patient [1]. Preoperative evaluation of the patient's neurological status involves analyzing cognitive function (level of consciousness, communication, and intellect), and language (ability to communicate in written and verbal form). Sensory evaluation, motor integration, and written or verbal communication reveal disorders in specific cortical areas.

It is difficult to get detailed history and information from patients with low consciousness level. In such cases, only families or medical records can be applied to obtain required information. Preoperative assessment should give priority to identification of neurological status and comorbidities. Neurologic history of the patient should be also analyzed and relevant medications, symptoms and lesions, if any, should be identified and recorded. Inquiries should aim at identifying symptoms of increased intracranial pressure, such as seizures, neurological deficits, headache, nausea, vomiting, and confusion, and obtaining the patient's history of transient ischemic attack or stroke. Afterwards, any change in the level of consciousness, symptoms and signs of increased intracranial pressure, sensory and motor deficits and seizures are recorded.

Preoperative neurological assessment includes evaluation of the functions of both peripheral and central nervous systems. The preoperative physical examination of other organ systems including pulmonary, cardiac, etc., should be done before the neurological examination. Thus, other physical findings can be integrated with the presenting neurological abnormality and it can be identified whether the primary disorder is related to the neurological system or not. All patients that will receive general anesthesia should undergo a short neurological assessment even if they do not have any visible neurological disorder. If a general or regional anesthesia technique affects an organ system, that system should be examined and findings should be recorded by the anesthesiologist in the preoperative period. In order to examine the musculoskeletal or motor system, physicians may observe the patient's strength, gait, and ability to walk on the toes, walk on the heels, and hold his/her arms in a specific position. Sensory system assessment includes testing for vibration sensation, pain sensation and light touch sensation on feet and hands of the patient. Superficial reflexes and deep-tendon reflexes can be analyzed easily and quickly. Cranial nerve disorders are searched out through patient's history and observation. Findings regarding the mental status of the patients, such as mood, appearance and cognitive functions, and the speech patterns are demonstrated during communication with the patient.

Neurological physical examination should include assessment of sensory and motor systems, and cranial nerves. Any improvement or worsening in neurological status of the patient can be monitored and identified through sequential examinations. Development of neurological deficits can be detected with repeated examinations [2]. AVPU and Glascow Coma Scale (GCS) are utilized to assess the conscious state of the patient [3]. Patient's state of consciousness, maintenance of airway patency, and cervical spine immobilization, especially in trauma patients, are important during the initial evaluation of the patient.

The GCS provides anesthesiologist with information regarding patient evaluation and the necessity of intubation. Sensory and motor functions should be briefly examined and sensory or motor deficits or weakness, if any, developing in the extremities should be recorded. Cranial nerve involvement or dysfunction may affect perioperative anesthetic management of the patient. Oculomotor nerve controls the pupil size and pupillary response to light. Preoperative size of the pupil should be recorded not only for intraoperative monitoring of the depth of anesthesia but also for comparison of changes that may develop in the pupil during the postoperative period. Patients with a GCS score of 13-15 are oriented patients. While a score of 8-12 indicates that the patient is in precoma period, a score $<7$ represents coma. Spontaneous eye opening is an indicative of brainstem activity. A score of 3 and 4 obtained from the first section shows that the cerebral cortex can process information.

Preoperative anxiety is common in neurosurgery patients. However, preoperative treatment with anxiolytic or opioids is controversial. Patients with symptoms of increased intracranial pressure are at increased risk of respiratory depression, and sedative agents can further increase the intracranial pressure in these patients. In general, if preoperative sedation will be applied in patients with an intracranial disease, they are recommended to receive sedation preferably with reversible sedatives and in an environment where the patient is monitored and their airway can be easily managed [4].

Disorders developing in 6 of the cranial nerves may affect patient management under anesthesia. The olfactory nerve is responsible for the sense of smell (the first head pair). Anosmia developing without any nasal disorder or inflammation may be a sign of frontal lobe or pituitary lesions, meningitis, hydrocephalus or fractures of the anterior fossa of the skull. Presence of increased intracranial pressure, cerebral hemorrhage, and meningeal signs of infection may require anesthesia. The oculomotor nerve controls the pupil size and pupillary response to light. Patients with trigeminal neuralgia often have unilateral 
symptoms limited to the $2^{\text {nd }}$ (maxillary) and $3^{\text {rd }}$ (mandibular) divisions of the nerve. Trigeminal neuralgia mostly results from the compression of the trigeminal nerve by the posterior fossa artery. Anesthesiologist can precipitate an attack of pain by touching the face mask upon the trigger zones around the lips or buccal cavity. Facial nerve innervates muscles of facial expression, achieves symmetry, and receives sensory innervation of taste sensation. The facial nerve may be damaged due to the pressure of mask ventilation, surgery or position. A record must be created depending on the baseline assessment performed prior to the induction of anesthesia. The glossopharyngeal nerve provides sensory innervation of the pharynx and soft palate mucosa. Glossopharyngeal injury causes severe pain similar to trigeminal neuralgia. The trigger point is in the posterior pharynx and tonsils. Stimulation of this point by oral airway or laryngoscopy may result in severe local pain radiating to the jaw or the ears. Moreover, reflex bradycardia and hypotension may occur during pain episodes in the glossopharyngeal nerve injury.

The vagus nerve gives motor fibers to the larynx, pharynx, trachea, and soft palate. Sensory and motor support is provided to the trachea and larynx through the superior and recurrent laryngeal branches of the vagus nerve. Hoarseness is an indicative of vocal cord paralysis resulting from recurrent laryngeal nerve injury. Tachycardia may be seen in the patients suffering from the injury of the vagus nerve.

There are three reflex types. These are deep-tendon, pathological and superficial reflexes. Deep-tendon reflexes can be easily examined using the biceps, patellar, and Achilles tendons. While examination of Achilles reflexes checks the S1 and S2 nerve roots, biceps reflexes check C5-6 and patellar reflexes check L2-3-4. When an object touches the skin, superficial reflexes enable identification of the segment functions of the Central Nervous System. Increased reflexes are observed in patients with hyperthyroidism and upper motor neuron disease. Decreased reflexes, in contrast, are observed in hypothyroidism. The most common pathological reflex is the Babinski sign.

Patients presenting with cerebrovascular occlusive disease, such as carotid artery or vertebrobasilar arterial disease, usually manifest signs or symptoms of either a transient ischemic attack or stroke. Uncontrolled systemic hypertension should be treated preoperatively without lowering blood pressure to a level which may precipitate ischemic symptoms. Since transient ischemic attacks are transient, patients may not show any TIA sign during examination.

Patients with cerebral infarction secondary to carotid artery occlusion may have symptoms of transient ischemic attack due to perioperative head and neck lesions. These patients usually have a history of severe cervical arthritis and spondylolisthesis. Careful head positioning is essential in the operating room to avoid precipitation of cerebral ischemia. As there is the risk of dislodging and embolizing a carotid plaque, the diseased carotid artery should not be palpated during examination.

The GCS provides instructive information for the anesthesiologist in patients undergoing craniotomy operation due to head trauma. Also, the GCS reveals the urgency of a patient's need for intubation and neurosurgical intervention. Seizures may accompany cerebral trauma and suggest the expansion of an intracranial hematoma. Airway management, ventilation and shock therapy must be the primary focus of anesthesia. Both hypertension and hypotension are manifestations of head injuries and require immediate treatment.

\section{References}

1. Schiavi A, Papangelou A, Mirski M (2009) Preoperative preparation of the surgical patient with neurologic disease. Med Clin North Am 93: 1123-1130.

2. Bledsoe BE, Porter RS, Shade BR (1997) Paramedic Emergency Care (3rdedn). Prentice Hall Inc, New Jersey, 749-754.

3. Matis G, Birbilis T (2008) The Glasgow Coma Scale--a brief review. Past, present, future. Acta Neurol Belg 108: 75-89.

4. Sivanaser V, Manninen P (2010) Preoperative assessment of adult patients for intracranial surgery. Anesthesiol Res Pract 2010. 